\title{
The application of a compound model for predicting reliability indices of engineering systems
}

\author{
A. Kudzys \\ *Member of the Lithuania Academy of Science, Gedimino 3, 01103 Vilnius, Lithuania, \\ **Vilnius Gediminas Technical University, J. Basanavičiaus 28, 03224 Vilnius, Lithuania, \\ E-mail: ona.lukoseviciene@vgtu.lt
}

cross $^{\text {ref }}$ http://dx.doi.org/10.5755/j01.mech.22.2.12268

\section{Introduction}

Engineering systems are widely used for performing technological tasks in energetics, industry, transport, farming and population areas. Reliability indices of engineering systems must be determined considering their analyzed reference period. The brittle fracture of the first component in a series system may cause a failure in this structure (Fig. 1, a). Thus, the system made of series connected components $m$ is at the stage of decomposition in case any of its components has failed. When the components of this system are ductile, it can continue to operate under reduced reliability indices of the components. A system (Fig. 1, b) consisting of perfectly ductile parallel connected components can fail only when all of its contributory units have reached their limit states [1]. Then, the strongest component $m$ of the ductile parallel system must survive in all extreme situations [2].

For static engineering systems, the safety or failure regions are determined referring to the unions and intersections of survival or failure probabilities of the number of ductile components, $m$, which depends on a number of random modes of discrete failure [1]. Therefore, dynamic reliability indices of auto-systems may be fined using failure modelling of their stochastically dependent components.

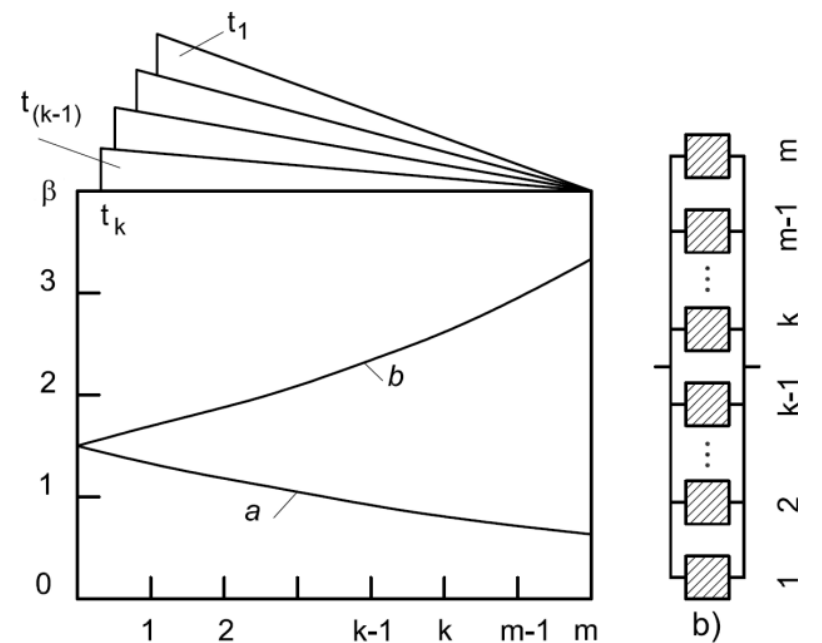

a)

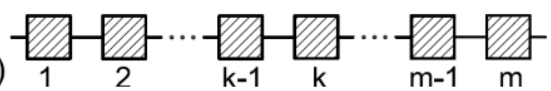

Fig. 1 Reliability index, $\beta$, versus the number of the components for series (a) and parallel (b) systems
The stochastic reliability parameters of an engineering system depend on recurrent extreme events that are likely to occur during its reference working life. In spite of the shot duration of extreme actions, they belong to persistent design situations characterized by random nonstationary safety margin processes. An objective prediction for reliability indices of engineering systems using deterministic and semi-probabilistic methods is hardly moved into engineering reality. Regardless of efforts to improve the semi-probabilistic formats, it is inconceivable to fix the correct values of the reliability indices of stochastic systems.

Any auto-system may be analyzed as a series system with highly correlated particular elements characterizing the behaviour of substantial elements at times $t_{1}, t_{2}, \ldots$, $t_{n}$.

Using fully probability-based approaches reliability indices may be predicted. Probability-based models allow us to explicitly define basic and additional uncertainties of the analyzed models. However, the probabilistic analysis of the systems involves the integration of multivariate and multistate distributions. The exact probabilitybased analysis using multidimensional integrals of the systems having stochastically dependent components is also very difficult.

Failure probabilities of series and parallel systems as the unions and intersections of these probabilities may be defined by equations:

$$
\begin{aligned}
& P\left(\bigcup_{k=1}^{m} F_{k}\right)_{s s}=P\left[\bigcup_{k=1}^{m}\left(X_{k} \leq-\beta_{k}\right)\right]_{s s} \approx 1-\Phi_{m}(-c, \rho) ; \\
& P\left(\bigcap_{k=1}^{m} F_{k}\right)_{p s}=P\left[\bigcap_{k=1}^{m}\left(X_{k} \leq \beta_{k}\right)\right]_{p s} \approx \Phi_{m}(c, \rho),
\end{aligned}
$$

where $m$ is the number of components or failure modes; $X_{k}$ $(k=1,2, \ldots, m)$ is a normal random variable; $\beta_{k}$ is a reliability index of the $k$-th component of the systems; $\rho$ is a quadratic matrix of correlation coefficients.

Computational methods for the probabilistic analysis of highly reliable systems are based on the variablecomplexity approach [3], importance and conditional sampling procedures [4], or direction simulation approaches [5]. However, these improvements are inconvenient by reason of mathematical complication.

Pandey and co'authors [6-10] proposed the simplified computational method for a product of conditional marginal (PCM). The computation of this method is simplified using the multidimensional normal distribution of 
the joint, which is expressed as a product of conditional probabilities $m$ approximating by one-dimensional normal distribution. When using the product of the conditional marginal (PCM) method, cumulative failure probabilities of series and parallel systems in failure modes may be expressed using Eqs. (1) and (2) as follows:

$$
\begin{aligned}
& P\left(\bigcup_{k=1}^{m} F_{k}\right)_{s s} \approx 1-\prod_{k=1}^{m} \Phi\left(c_{k \mid(k-1)}\right) ; \\
& P\left(\bigcap_{k=1}^{m} F_{k}\right)_{p s} \approx \prod_{k=1}^{m} \Phi\left(c_{k \mid(k-1)}\right),
\end{aligned}
$$

where $c_{k \mid(k-1)}$ is a conditional normal fractile defined under the conditional variable $X_{k \mid(k-1)}=X_{k} \bigcap_{i=1}^{(k-1)} X_{i} \leq c_{i} \quad$ which is normally distributed.

Based on the principles of conditional probability and the theoretical expression of equivalent linear failure modes (ELFM), an approximate method has been proposed by Kang et al. [11]. This method is suitable for calculating failure probabilities taking into account series and parallel systems.

Li et al. [12] suggested approaches providing a possibility of transforming the computation of the probability of the system usually leading to the multidimensional integration of joint probability density functions and to the one-dimensional integration of the probability density functions of the equivalent extreme-value.

Based on simplified matrix procedures, the matrix-based system reliability (MSR) method introduced by Song and Kang [13] may be used for calculating failure probabilities in the system.

Kang and Song [14] suggested the method developed for evaluating multivariate normal integrals defined for a general system of the events, including series, parallel, cut-set and link-set systems. This method leads to making a compound of two components sequentially coupled by the union or intersection until the systems become a single compound event. Numerical procedures are developed to obtain the reliability index of a new compound event. However, analyses of systems consisting of not equireliable and not equicorrelated components is difficult.

This paper is aimed to show a uniform compound methodology based on the probabilistic concepts for reliability assessment of engineering systems. The compound methodology recommends to separate of dynamic (timedependent) probabilistic models of auto-systems from the static models of general engineering systems. For assessing survival probabilities of auto-systems affected by random demands $n$ and general systems comprised of correlated auto-systems $m$, the concept of the method for transformed conditional probabilities (TCP) and conventional correlation vectors $(\mathrm{CCV})$ may be used.

\section{Auto-systems as the components of general systems}

\subsection{Dynamic stochastic parameters}

Predictions for dynamic (time-dependent) survival probabilities of auto-systems are very relevant in the probabilistic analysis of engineering systems.
The time-dependent safety margin or limit state function of perfectly ductile particular element cut $t_{k}$ of the auto-system may be defined as:

$$
Z_{k}(t)=g\left(\boldsymbol{X}_{k}(t), \boldsymbol{\theta}\right)=g\left(\theta_{R} R-\boldsymbol{\theta}_{E} \boldsymbol{E}_{k}(t)\right),
$$

where $\boldsymbol{X}_{k}(t)$ and $\boldsymbol{\theta}$ are the vectors of basic and additional variables; $R$ (response) and $\boldsymbol{E}_{k}(t)$ (demands) are random resistance and action effects of particular elements; $\theta_{R}$ and $\boldsymbol{\theta}_{E}$ are uncertainties of the design model. For analyzing the auto-system, a finite decreasing sequence and survival probabilities $\quad P\left[S_{1}(t)\right] \geq P\left[S_{2}(t)\right] \geq \ldots \geq P\left[S_{k}(t)\right] \geq \ldots$ $\ldots \geq P\left[S_{n}(t)\right]$ are used.

The concepts of transformed conditional probabilities (TCP) and conventional correlation vectors (CCV) have been proposed by Kudzys and co-authors $[1,15,16,17]$. According to these concepts, transition probability of a failure in the ductile auto-system at time $t_{k}$, assuming that the analyzed element was safe at time less than $t_{k}$, may be expressed by the equation:

$$
\begin{aligned}
& P\left[F_{k \mid 1}(t)\right]=P\left[F_{k}(t) \mid \bigcap_{i=1}^{(k-1)} S_{i}(t)\right] \approx \\
& \approx 1-P\left[S_{k}(t)\right] \times\left(1+\rho_{k \mid f}^{x_{k}(t)}(t)\left[\frac{1}{P\left[S_{k}(t)\right]}-1\right]\right),
\end{aligned}
$$

where $F_{k}(t)$ and $S_{k}(t)$ are the failure and survival probabilities of single particular elements at time $t_{k}$.

The conventional correlation factor in stochastically dependent sequence cuts is formed from the $k$-th row of the quadratic matrix of the basic coefficients of correlation. Therefore, a conventional correlation factor may be expressed as follows:

$$
\rho_{k \mid f}(t)=\frac{1}{k-1} \sum_{i=1}^{(k-1)} \rho_{k i}(t) .
$$

Thus, for practical sake, the matrix of the correlation coefficient may be modified using the conventional correlation vector $(\mathrm{CCV})$ consisting of bounded correlation factors written in the form:

$$
\boldsymbol{\rho}=\left[1, \rho_{2 \mid 1}^{x_{2}(t)}(t), \ldots, \rho_{k \mid 1 \ldots(k-1)}^{x_{k}(t)}(t)\right]^{T} .
$$

The correlation coefficients of the elements or sequence cuts of the auto-system may be expressed as follows:

$$
\rho_{k i}(t)=\rho\left[Z_{k}(t), Z_{i}(t)\right]=\frac{\operatorname{Cov}\left[Z_{k}(t), Z_{i}(t)\right]}{\sigma\left[Z_{k}(t)\right] \times \sigma\left[Z_{i}(t)\right]},(9)
$$

where $\operatorname{Cov}\left[Z_{k}(t), Z_{i}(t)\right]$ and $\sigma\left[Z_{k}(t)\right]$ and $\sigma\left[Z_{i}(t)\right]$ are auto covariance and standard deviations from safety margins $Z_{k}(t)$ and $Z_{i}(t)$ computed using Eq. (5).

The bounded index of a conventional correlation factor in the random multi-cut sequence may be defined as follows: 


$$
x_{k}(t)=P\left[S_{1}(t)\right] \times\left(\frac{4.5+4 \times \rho_{k f}(t)}{1-0.98 \times \rho_{k f}(t)}\right)^{v_{k}(t)},
$$

where its index

$$
\begin{aligned}
& v_{k}(t)=\frac{\sum_{i=2}^{k} P\left[S_{i}(t)\right]}{(k-1)\left[k+2 \beta_{k}^{2}(t)-15 \beta_{k}(t)+30\right]^{1 / 2}}, \\
& P_{a s}(S)=P_{a s}\left[\bigcap_{k=1}^{n} S_{k}(t), \exists S_{(k-1)}(t) \geq S_{k}(t), k \in(1, n)\right] \approx 1-\left[\begin{array}{l}
P\left[F_{1}(t)\right] \bigcup P\left[F_{2}(t) \mid S_{1}(t)\right] \bigcup \ldots \\
\ldots \cup P\left[F_{k}(t) \mid \bigcap_{i=1}^{(k-1)} S_{i}(t)\right] \bigcup \ldots \\
\ldots \cup P\left[F_{n}(t) \mid \bigcap_{i=1}^{(n-1)} S_{i}(t)\right]
\end{array}\right] \approx P\left[S_{1}(t)\right] \times \\
& \times P\left[S_{2}(t)\right] \times\left\{1+\rho_{2 \mid f}^{x_{2}(t)}(t)\left[\frac{1}{P\left[S_{1}(t)\right]}-1\right]\right\} \times \ldots \times P\left[S_{k}(t)\right] \times\left\{1+\rho_{k \mid f}^{x_{k}(t)}(t)\left[\frac{1}{P\left[S_{k-1}(t)\right]}-1\right]\right\} \times \ldots \times \\
& \times P\left[S_{n}(t)\right] \times\left\{1+\rho_{n \mid f}^{x_{n}(t)}(t)\left[\frac{1}{P\left[S_{n-1}(t)\right]}-1\right]\right\} \approx P\left[S_{1}(t)\right] \times \prod_{k=2}^{n}\left(P\left[S_{k}(t)\right]\left\{1+\rho_{k \mid f}^{x_{k}(t)}(t)\left[\frac{1}{P\left[S_{k-1}(t)\right]}-1\right]\right\}\right)
\end{aligned}
$$

where $n$ is the number of the extreme situations of the nonstationary demand process and $\rho_{k \mid f}^{x_{k}(t)}(t)$ is the indexed conventional correlation factor that may be defined using Eqs. (7) and (10).

\section{Series and parallel general engineering systems}

\subsection{Static survival probabilities}

The safety margins of the components (autosystems) of static general engineering systems may be defined as:

$$
Z_{k}=g\left(\boldsymbol{X}_{k}, \boldsymbol{\theta}\right)=g\left(\theta_{R} R-\boldsymbol{\theta}_{E} \boldsymbol{E}_{k}\right),
$$

where $k=1,2, \ldots, m$ depends on the $k$-th random discrete failure mode of engineering systems at their service time.

Following the same probabilistic approaches and analogically to Eq. (12), cumulative survival probability for static series ductile general systems (Fig. 1, a) may be expressed as:

$$
\begin{aligned}
& P_{s s}(S)=P_{s s}\left[\bigcap_{k=1}^{m} S_{k}, \exists P\left(S_{k}\right) \geq P\left(S_{(k-1)}\right), k \in(1, m)\right]= \\
& =1-P_{s s}\left(\bigcup_{k=1}^{m} F_{k}\right) \approx \\
& \approx P\left(S_{1}\right) \times \prod_{k=2}^{m}\left(P\left(S_{k}\right) \times\left\{1+\rho_{k \mid f}^{x_{k}}\left[\frac{1}{P\left(S_{k}\right)}-1\right]\right\},\right.
\end{aligned}
$$

where $P\left(S_{k}\right)$ is the survival probability of $k$-th ductile autosystems; $m$ is the number of components or failure modes of engineering systems; $\rho_{k \mid f}^{x_{k}}$ is the indexed conventional correlation factor, the value of which is defined using Eqs. (7) and (10) and correlation coefficients $\rho_{k i}=\rho\left(Z_{k}, Z_{i}\right)=\operatorname{Cov}\left(Z_{k}, Z_{i}\right) /\left(\sigma Z_{k} \times \sigma Z_{i}\right)$ of the quadratic matrix of correlation coefficients.

Cumulative survival probability of static parallel ductile engineering systems (Fig. 1, b) consists of components $m$ may be expressed as:

$$
\begin{aligned}
& P_{p s}(S)= \\
& =1-P_{p s}\left[\bigcap_{k=1}^{m} F_{k}, \exists P\left(F_{k}\right) \leq P\left(F_{(k-1)}\right), k \in(1, m)\right] \approx \\
& \approx 1-P\left(F_{1}\right) \times \prod_{k=2}^{m}\left(P\left(F_{k}\right) \times\left\{1+\rho_{k \mid f}^{x_{k}}\left[\frac{1}{P\left(F_{k}\right)}-1\right]\right\},\right.
\end{aligned}
$$

where $P\left(F_{k}\right)$ is the failure probability of the $k$-th ductile auto-system of the sequence of failure probabilities $P\left(F_{l}\right) \geq \ldots \geq P\left(F_{k}\right) \geq \ldots \geq P\left(F_{m}\right)$. For parallel general systems, the demand vector $\boldsymbol{\theta}_{E} \boldsymbol{E}_{k}$ corresponds to failure state after statical redistribution of actions. When the correlation factor by Eq. (7) is equal to 0 or 1, the suggested Eqs. (12), (14) and (15) give the exact solutions.

Series and parallel engineering systems may be introduced by generalized reliability indices:

$$
\begin{aligned}
& \beta_{s s}=\Phi^{-1}\left[P_{s s}(S)\right], \\
& \beta_{p s}=\Phi^{-1}\left[P_{p s}(S)\right],
\end{aligned}
$$

where $\Phi[\bullet]$ is the cumulative distribution function of the 
standard normal distribution; $P_{s s}(S)$ and $P_{p s}(S)$ are survival probabilities of series and parallel engineering systems calculated using Eq. (14) and Eq. (15), respectively.

\subsection{Target reliability indices}

The selection of rational minimum values of target reliability indices, $\beta_{S T}$, of different type general engineering systems requires special investigations. However, their optimum values should be always related to working capacity reserves of the injured systems and functional working classes of their components in critical demand situations. When the injured elements of the systems are not easily repairable and replaceable, the system belongs to classes FC1 and FC2, respectively. The systems with not repairable, and not replaceable components may be ascribed to FC3 class.

The target reliability index, $\beta_{S T}$, of general engineering systems depends on human life, economic, social and environmental consequences due to their failure. However, differentiation and classification processes of $\beta_{S T}$ of the systems are closely related not only to the consequences of their failure but also to incremental reliability costs. When the expected loss of human life is significant, it is very difficult to choose the optimal value of target reliability index, $\beta_{S T}$, because to estimate the material losses per human life loses is very intricate.

When the additional reliability of engineering systems can be achieved at a low cost, then larger target reliability index, $\beta_{S T}$, may be acceptable [18]. Any system having equally important failure modes should be designed for a higher level of $\beta_{S T}$. The smallest and largest values of $\beta_{S T}$ belong to information processing systems and dangerous construction works, respectively.

Some possible values of target reliability index, $\beta_{S T}$, are presented in Table 1 . The proposed recommendations may satisfy the agreements between reliability and economy factors in engineering systems.

The design documents $[19,20]$ and recommendations by the JCSS [21] are used for designing construction work members and their systems. An overview of these documents and their reliability classes RC1, RC2 and RC3 are associated with consequence classes $\mathrm{CC} 1, \mathrm{CC} 2$ and CC3 when the target values of reliability indices, for the period of 50-year reference, have been equal to $\beta_{S T}=3.3-4.3$.

Table 1

Target reliability indices of engineering systems

\begin{tabular}{|c|c|c|c|c|c|c|}
\hline \multirow{3}{*}{ 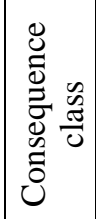 } & \multicolumn{6}{|c|}{ Functional working class } \\
\hline & \multicolumn{3}{|c|}{$\begin{array}{l}\text { For } 20 \text { years reference period } \\
\text { of equipment's }\end{array}$} & \multicolumn{3}{|c|}{$\begin{array}{l}\text { For } 50 \text { years refer- } \\
\text { ence period of con- } \\
\text { struction works }\end{array}$} \\
\hline & $\mathrm{FC} 1$ & $\mathrm{FC} 2$ & FC3 & $\mathrm{FC} 1$ & $\mathrm{FC} 2$ & FC3 \\
\hline $\mathrm{CC} 1$ & $0.5-1.0$ & $0.75-1.25$ & $1.0-1.5$ & 3.1 & 3.3 & 3.8 \\
\hline $\mathrm{CC} 2$ & $0.75-1.25$ & $1.0-1.5$ & $1.25-1.75$ & 3.3 & 3.8 & 4.3 \\
\hline CC3 & $1.0-1.5$ & $1.25-1.75$ & $1.5-2.0$ & 3.8 & 4.3 & 4.7 \\
\hline
\end{tabular}

Particular elements and structural members of the structure may be designed for the same higher or lower reliability index as for the entire system [1]. The difference between reliability indices of the systems of the events and their components should depend on the coefficient of correlation $\rho_{k i}$ of these components. When the correlation coefficient of series and parallel systems increases, target reliability index, $\beta_{S T}$, may increase and decrease [18].

\section{Numerical illustrations}

\subsection{Series auto-systems consist of component $n$}

The series auto-systems consisting of $n$ equireliable and equicorrelated components have been analysed $[8,11]$. There are presented several cases when reliability indices, $\beta_{k}(t)$, of each component of the system is equal to 3.5 and 4.0. The initial data of auto-system and results of calculation are shown in Table 2 . The reliability indices of series auto-systems are computed using three different computational methods.

Table 2

Reliability indices of series auto-systems

\begin{tabular}{|c|c|c|c|c|c|}
\hline \multirow{2}{*}{$\begin{array}{l}\text { Reliability index } \\
\text { of component }\end{array}$} & \multirow{2}{*}{$\begin{array}{c}\text { The number } \\
\text { of components, } \\
n\end{array}$} & \multirow{2}{*}{$\begin{array}{l}\text { Conventional } \\
\text { correlation } \\
\text { factor } \rho_{k i}(t)\end{array}$} & \multicolumn{3}{|c|}{ Design methods } \\
\hline & & & $\begin{array}{c}\text { TCP+CCV by } \\
\text { Eq. (16) }\end{array}$ & $\begin{array}{l}\text { Numerical integ- } \\
\text { ration [8] }\end{array}$ & PCM [8] \\
\hline \multirow{8}{*}{3.5} & \multirow{4}{*}{7} & 0.30 & 2.960 & 2.948 & 2.945 \\
\hline & & 0.50 & 2.984 & 2.968 & 2.949 \\
\hline & & 0.70 & 3.028 & 3.022 & 2.953 \\
\hline & & 0.90 & 3.138 & 3.164 & 2.960 \\
\hline & \multirow{4}{*}{27} & 0.30 & 2.541 & 2.521 & 2.509 \\
\hline & & 0.50 & 2.594 & 2.584 & 2.526 \\
\hline & & 0.70 & 2.680 & 2.711 & 2.547 \\
\hline & & 0.90 & 2.876 & 2.976 & 2.572 \\
\hline \multirow{7}{*}{4.0} & \multirow{2}{*}{5} & 0.10 & 3.603 & 3.601 & 3.601 \\
\hline & & 0.95 & 3.792 & 3.790 & 3.604 \\
\hline & \multirow{3}{*}{20} & 0.10 & 3.230 & 3.224 & 3.224 \\
\hline & & 0.50 & 3.293 & 3.254 & 3.227 \\
\hline & & 0.95 & 3.293 & 3.254 & 3.227 \\
\hline & \multirow{2}{*}{50} & 0.50 & 3.057 & 3.016 & 2.962 \\
\hline & & 0.90 & 3.346 & 3.426 & 2.981 \\
\hline
\end{tabular}


Illustrative example shows that the $\mathrm{TCP}+\mathrm{CCV}$ concepts may help us to predict reliability indices of the series auto-system and estimate their quantitative durability level.

\subsection{Failure probabilities and reliability indices of engineering systems}

The values of failure probabilities and reliability indices of series and parallel engineering systems consisting of six equireliable components have been analyzed. The reliability index of these components in the engineering system is equal to $\beta=1-3[6,7]$.

The quadratic matrix of the basic coefficients of correlation may be written as follows:

$$
\boldsymbol{\rho}_{k i}=\left|\begin{array}{cccccc}
1 & 0.8292 & 0.7312 & 0.6180 & 0.4787 & 0.2764 \\
0.8292 & 1 & 0.6614 & 0.5590 & 0.4330 & 0.2500 \\
0.7313 & 0.6614 & 1 & 0.4931 & 0.3819 & 0.2205 \\
0.6180 & 0.5590 & 0.4931 & 1 & 0.3227 & 0.1863 \\
0.4787 & 0.4330 & 0.3819 & 0.3227 & 1 & 0.1443 \\
0.2764 & 0.2500 & 0.2205 & 0.1863 & 0.1443 & 1
\end{array}\right|
$$

Conventional correlation factors may be computed using the quadratic matrix of the basic coefficients of correlation and Eq. (7). Then, conventional correlation factors in the engineering systems are equal to $\rho_{2 \mid 1}=0.8292$, $\rho_{3 \mid 21}=0.6963, \quad \rho_{4 \mid 321}=0.5567, \quad \rho_{5 \mid 4321}=0.4041 \quad$ and $\rho_{6 \mid 54321}=0.2155$.

The failure probabilities and reliability indices of series and parallel systems with six equireliable components are calculated by different methods. Calculation results are presented in Tables 3 and 4.

Table 3

Failure probabilities and reliability indices of series engineering systems having 6 equireliable components

\begin{tabular}{|c|c|c|c|}
\hline \multirow{2}{*}{$\beta_{e l}$} & \begin{tabular}{c} 
DCP+CCV \\
by \\
\cline { 2 - 4 }
\end{tabular} & $\begin{array}{c}\text { Numerical Inte- } \\
\text { gration } \\
\text { by Eq.(1) [6, 7] },(16)\end{array}$ & $\begin{array}{c}\text { PCM } \\
\text { by Eq.(3) [6, 7] }\end{array}$ \\
\hline 3 & $\frac{0.006694}{2.47}$ & $\frac{0.006928}{2.46}$ & $\frac{0.007675}{2.42}$ \\
\hline 2 & $\frac{0.09734}{1.30}$ & $\frac{0.09544}{1.31}$ & $\frac{0.1012}{1.28}$ \\
\hline 1 & $\frac{0.4708}{0.07}$ & $\frac{0.4665}{0.08}$ & $\frac{0.4641}{0.09}$ \\
\hline
\end{tabular}

Table 4

Failure probabilities and reliability indices of parallel engineering systems having 6 equireliable components

\begin{tabular}{|c|c|c|c|}
\hline \multirow[b]{2}{*}{$\beta_{e l}$} & \multicolumn{3}{|c|}{ Design methods } \\
\hline & $\begin{array}{c}\mathrm{TCP}+\mathrm{CCM} \\
\text { by } \\
\text { Eq.(15),(17) }\end{array}$ & $\begin{array}{l}\text { Numerical In- } \\
\text { tegration } \\
\text { by Eq.(2) }[6,7]\end{array}$ & $\begin{array}{c}\text { PCM } \\
\text { by Eq. (4) }[6,7\end{array}$ \\
\hline 3 & $\frac{20.13 * 10^{-8}}{5.07}$ & $\frac{7.035^{*} 10^{-8}}{5.26}$ & $\frac{4.556^{*} 10^{-8}}{5.34}$ \\
\hline 2 & $\frac{3.740 * 10^{-5}}{3.96}$ & $\frac{5.282 * 10^{-5}}{3.88}$ & $\frac{4.390 * 10^{-5}}{3.92}$ \\
\hline 1 & $\frac{5.264 * 10^{-3}}{2.56}$ & $\frac{5.926 * 10^{-3}}{2.52}$ & $\frac{5.829 * 10^{-3}}{2.52}$ \\
\hline
\end{tabular}

Failure probabilities and reliability indices are calculated using the TCP+CCV method by Eq. (14) and Eq. (16) for series and by Eq. (15) and Eq. (17) for parallel systems.

In illustrative examples presented results of survival and failure probabilities and reliability indices of the engineering system calculated using the proposed method of transformed conditional probabilities with conventional correlation vectors $(\mathrm{TCP}+\mathrm{CCV})$, the method of the product of conditional marginal (PCM) and exact numerical integration are very close.

\section{Conclusions}

The proposed concept of the compound design model and refusing complex multidimensional integrals helps us with avoiding mathematical problems of the prediction analysis of dynamic (time-dependent) reliability indices of engineering systems. For calculating the probability of dynamics, series auto-systems consisting of particular element cuts $n$ and static series and parallel general systems made of correlated auto-systems $m$ are used for the compound design model.

The formulated concepts of transformed conditional probabilities (TCP) with conventional correlation vectors $(\mathrm{CCV})$ allow us to predict reliability indices of series and parallel engineering systems.

The suggested compound methodology of reliability analysis of engineering systems using dynamic and static stages helps designers with conducting probabilitybased analysis in design practice. The conducted unsophisticated probabilistic analysis of design models may stimulate designers to use probabilistic methods in their practice in a more efficient and active way.

\section{Acknowledgements}

The second author gratitude to the Research Council of Lithuania for the Postdoctoral fellowship. Postdoctoral fellowship is being funded by European Union Structural Funds project "Postdoctoral Fellowship Implementation in Lithuania" within the framework of the Measure for Enhancing Mobility of Scholars and Other Researchers and the Promotion of Student Research (VP1-3.1ŠMM-01) of the Program of Human Resources Development Action Plan.

\section{References}

1. Lukoševičienė, O.; Kudzys, A. 2014. Conventional correlation factors in the safety analysis of stochastic systems. Safety and Reliability: Methodology and Applications contains the proceedings of the 24th European Safety and Reliability Conference, ESREL 2014, Wroclaw, Poland, 14-18 September 2014, 1741-1747. http://dx.doi.org/10.1201/b17399-236.

2. Dey, A.; Mahadevan, S. 2000. Reliability estimation with time-variant loads and resistances, Journal of Structural Engineering 126(5): 612-620. http://dx.doi.org/doi:10.1061/(ASCE)07339445(2000)126:5(612).

3. Burton, S.A., Hajela, P. 2003. A variable-complexity approach to second-order reliability-based optimization, Structural and Multidisciplinary Optimization 
25: 237-250.

http://dx.doi.org/10.1007/s00158-003-0298-3.

4. Mori, Y.; Ellingwood, B.R. 1993. Time-dependent system reliability analysis by adaptive importance sampling. Structural Safety 12(1): 59-73. http://dx.doi.org/doi:10.1016/0167-4730(93)90018-V.

5. Ditlevsen, O. 1997. Structural reliability codes for probabilistic design - a debate paper based on elementary reliability and decision analysis concepts, Structural Safety 19(3): 253-270. http://dx.doi.org/doi: 10.1016/S0167-4730(97)00009-X

6. Pandey, M.D. 1998. A simple approach to multinormal integration with applications to the system reliability computation, Civil Engineering and Environmental system 15: 89-105. http://dx.doi.org/doi:10.1080/02630259808970232.

7. Pandey, M.D. 1998. An effective approximation to evaluate multinormal integrals, Structural Safety 20: 51-67. http://dx.doi.org/doi:10.1016/S0167-4730(97)00023-4.

8. An, Y.; Pandey, M.D. 2002. Evaluation of an approximate method for multinormal integration, The 4th Structural Specialty Conference of the Canadian Society for Civil Engineering, June 5-8, 2002. p. 10. http://dx.doi.org/doi:10.1007/s00158-003-0298-3.

9. Pandey, M.D.; Sarkar, A. 2002. Comparison of a simple approximation for multinormal integration with an importance sampling-based simulation method, Probabilistic Engineering Mechanics 17(2): 215-218. http://dx.doi.org/doi:10.1016/S0266-8920(02)00003-6.

10. Yuan, X.X.; Pandey, M. D. 2006. Analysis of approximations for multinormal integration in system reliability computation, Structural Safety 28(4): 361-377. http://dx.doi.org/doi:10.1016/j.strusafe.2005.10.002.

11. Kang, H.; Zhang, X., Wang, F. 2003. A reliability analysis method of offshore platforms, Proceedings of the thirteenth International offshore and polar engineering conference Honolulu, Hawaii, USA, May 25-30, 2003, 469-472.

12. Li, J.; Chen J.B.; Fan W.L. 2007. The Equivalent extreme-value event and evaluation of the structural system reliability, Structural Safety 29(2): 112-131. http://dx.doi.org/doi:10.1016/j.strusafe.2006.03.002.

13.Song, J.; Kang, W.H. 2009. System reliability and sensitivity under statistical dependence by matrix-based system reliability method, Structural Safety 31(2): 148156. http://dx.doi.org/doi:10.1016/j.strusafe.2008.06.012.

14. Kang, W.H.; Song, J. 2010. Evaluation of multivariate normal integrals for general systems by sequential compounding, Structural Safety 32(1): 35-41. http://dx.doi.org/doi:10.1016/j.strusafe.2009.06.001.

15. Kudzys, A.; Lukoševičienè O. 2009. On the safety prediction of deteriorating structures, Mechanika 78(4): 5-11.
16. Kudzys, A.; Kliukas R. 2010. Probability-based design of spun concrete beam-columns, Journal of Civil Engineering and Management 16(4): 451-461. http://dx.doi.org/doi:10.3846/jcem.2010.51.

17. Kudzys, A.; Lukoševičienė, O. 2013. Conventional stochastic sequences in reliability assessments and predictions of structural members, 11th International Conference on Modern Building Materials, Structures and Techniques, MBMST 2013, Procedia Engineering 57: 642-650. http://dx.doi.org/10.1016/j.proeng.2013.04.081.

18. Nowak A.S.; Szerszen 2003. Calibration of design code: for buildings (ACI 318): Part 1 statistical models for resistance, ACI Structural Journal 100(3): 377-391. http://dx.doi.org/doi:10.1016/S0167-4730(97)00023-4.

19. EN 1990. 2002. Eurocode: Basic of Structural Design.

20. ISO 2394. 1998. General Principles on Reliability for Structures.

21. JCSS 2000. Probabilistic Model Code: Part 1- Basis of Design.

A. Kudzys, O. Lukoševičienė

\section{THE APPLICATION OF A COMPOUND MODEL FOR PREDICTING RELIABILITY INDICES OF ENGINEERING SYSTEMS}

S u m m a r y

The article considers sophisticated formats of the multivariate integration of time-dependent technical reliability prediction for ductile series and parallel engineering systems as a structure embracing stochastically dependent components. The paper recommends the rational avoidance of complicated multidimensional integrals and the separation of dynamic (time-dependent) probabilistic models of auto-systems from the static models of general engineering systems. For assessing survival probabilities of autosystems affected by random demands $n$ and general systems comprised of correlated auto-systems $m$, the concept of the method for transformed conditional probabilities (TCP) and conventional correlation vectors (CCV) have been used. The article also discusses target reliability indices of engineering systems. The analysis of reliability indices to series and parallel engineering systems is demonstrated applying the numerical examples.

Keywords: survival probability, reliability index, engineering systems.

Received May 15, 2015

Accepted March 15, 2016 